\title{
Relación de la dieta con el color y la terneza de la carne vacuna
}

\author{
Della Rosa, M.M. ${ }^{1,2}$; Papaleo Mazzucco, J.' y Aello, M.S.'
}

'Unidad integrada Facultad de Ciencias Agrarias (Universidad Nacional de Mar del Plata) y Estación Experimental Agropecuaria Balcarce (INTA). Balcarce. Buenos Aires. Argentina.

${ }^{2}$ Consejo Nacional de Investigaciones Científicas y Técnicas (CONICET). Ciudad Autónoma de Buenos Aires. Argentina.

\section{PALABRAS CLAVE ADICIONALES}

Calidad sensorial.

Concentrados energéticos.

Forrajes.

Glucógeno.

\section{RESUMEN}

Para el consumidor de carne vacuna la calidad sensorial es importante al momento de la compra y el consumo. El color depende mayormente de la concentración de mioglobina y el pH, en tanto que la terneza está relacionada con el pH, la composición del colágeno y la degradación miofibrilar. Todos ellos pueden influenciarse por la dieta. La concentración de mioglobina depende del tipo de fibra muscular, cuyo metabolismo está influenciado por la dieta que reciban los animales. Las dietas de baja energía se asocian a fibras rojas, con metabolismo oxidativo y mayor concentración de mioglobina, mientras que las dietas ricas en energía estimulan el desarrollo de fibras glucolíticas. Estas últimas, tienen la capacidad de acumular más glucógeno. El metabolismo de las miofibras también afecta al contenido de enzimas proteolíticas, las fibras oxidativas presentan mayor concentración de calpaínas que las fibras glicolíticas. La estructura del colágeno es influenciada por la dieta de forma indirecta, ya que su efecto está mediado por la tasa de ganancia de peso. Al consumir dietas energéticas los animales incrementan la velocidad de ganancia de peso, lo que disminuye el colágeno total por un efecto de dilución debido al aumento de miofibrillas. Por otro lado, la energía puede retrasar la formación y estabilización de las estructuras del colágeno, con aumento de la fracción soluble. La dieta cumple un rol importante sobre la calidad de la carne, por lo tanto mantener un buen nivel de alimentación permite maximizar la calidad y lograr un producto con atributos sensoriales coincidentes con los requeridos por un gran número de consumidores.

\section{Relationship between animal diet with color and tenderness beef cattle}

\section{SUMMARY}

The sensorial quality of meat is important when consumers both purchase and eat it. When buying, color is most important, whereas tenderness is significant when eating. The color of the meat mostly depends on the concentration of myoglobin and $\mathrm{pH}$, while tenderness is related to $\mathrm{pH}$, collagen composition and myofibrillar degradation. Muscle fiber metabolism depends on the diet that the animals receive. Low-energy diets are associated with red fibers, oxidative metabolism, and increased myoglobin concentration. High-energy diets stimulate the development of glycolytic fibers, which have the ability to accumulate more glycogen. Myofiber metabolism also affects the content of proteolytic enzymes; oxidative fibers present a higher calpain concentration than glycolytic fibers. Collagen structure is affected by diet indirectly; its effect is mediated by daily weight gain. When consuming energetic diets, animals rapidly increase their weight, which will reduce total collagen by a dilution established by myofibrils increase. Additionally, an energetic diet can delay formation and stabilization of collagen structures, with an increasing soluble proportion. As diet plays an important role in the quality of meat, a good nutritional level allows maximizing quality; as a result, a large number of consumers will have a product with the sensory characteristics required.

\section{INFORMATION}

\section{Cronología del artíiculo.}

Recibido/Received: 07.09.2016

Aceptado/Accepted: 11.03.2017

On-line: 15.07 .2017

Correspondencia a los autores/Contact e-mail:

mm.dellarosa@gmail.com

\section{INTRODUCCIÓN}

La calidad sensorial puede definirse como el conjunto de características percibidas por los sentidos de una persona en el momento de la compra o del consumo, que influyen en la satisfacción sensorial (Troy y Kerry, 2010). Son múltiples las variables tenidas en cuenta por el consumidor para la compra de carnes 
rojas, que abarcan desde la capacidad adquisitiva y el sexo de la persona hasta la grasa visible y el grado de preparación de la carne, entre otros (Resurrección, 2004). No obstante, el color es el principal atributo de calidad que los consumidores utilizan al momento de realizar la compra, ya que lo relacionan directamente con el estado de frescura y la salubridad de la carne (Smith et al., 2000; Killinger et al., 2004a; Troy y Kerry, 2010). En cambio, la terneza, jugosidad, flavor y palatabilidad son los principales aspectos que se relacionan a la calidad durante su consumo (Troy y Kerry, 2010), siendo la terneza el atributo más influyente (Lusk, et al., 2001; Shackelford, et al., 2001).

Las modificaciones en la dieta, especialmente durante la etapa de terminación o engorde de los animales, afectan la calidad de la carne. Toda variación en la dieta que implique cambios en la tasa de ganancia de peso, modificación del metabolismo de las fibras musculares o, una vez faenado el animal, genere cambios en la relación entre el descenso del $\mathrm{pH}$ y la temperatura, afectará el color y/o la terneza.

Esta revisión se centrará en describir la influencia de la dieta animal sobre variables asociadas al tejido muscular, conectivo y graso que determinan el color y la terneza de la carne.

\section{MIOGLOBINA Y TIPO DE FIBRAS MUSCULARES}

\section{GeNERALIDADES}

La concentración de mioglobina está asociada con el color rojo, medido objetivamente a través del parámetro a* (Bidner et al., 1986; Muir et al., 1998; Kim et al., 2010). A medida que los animales avanzan en edad la concentración de mioglobina en el músculo se incrementa para compensar su pérdida de afinidad por el oxígeno (Seideman et al., 1984).

En cuanto al tipo de fibras musculares, en la carne existen las llamadas fibras rojas, blancas e intermedias. Las fibras rojas están más vascularizadas y poseen mayor capacidad oxidativa, debido a un mayor contenido de mitocondrias y mioglobina, en comparación con las fibras blancas que tienen metabolismo glicolítico (Ryu y Kim, 2005; Popp et al., 2015; Listrat et al., 2016). Las fibras intermedias, como su nombre lo indica, tienen metabolismo oxidativo-glicolítico. Las miofibras son muy plásticas y su actividad metabólica e hipertrofia depende de la actividad física, sexo, hormonas presentes en el animal y el tipo de dieta que recibe (Lefaucheur, 2010; Joo et al., 2013).

\section{EFECTO DE LA DIETA}

Vestergaard et al. (2000) y Priolo et al. (2001) atribuyen el color más oscuro de la carne de animales alimentados con dietas basadas en forrajes a una mayor cantidad de fibras rojas. Investigaciones más recientes de Shibata et al. (2014) sugieren que, en condiciones de pastoreo, el cambio metabólico de fibras glicolíticas a oxidativas ocurre por la acumulación de distintos metabolitos en el músculo esquelético, lo cual se debe tanto a la dieta como al e que realizan los animales durante el pastoreo.

Bidner et al. (1986) encontraron que los animales alimentados con granos presentaron menor concentración de mioglobina que aquellos alimentados con forrajes (2,9 vs. 3,5 mg/g, respectivamente). A su vez, Gatellier et al. (2005) observaron, en tres categorías de bovinos, un efecto de la dieta sobre el contenido de mioglobina, medido mediante la concentración de hierro hemínico ( $\mu \mathrm{g}$ de hierro por g de carne; ppm). En las vaquillonas y vacas, el nivel de hierro hemínico fue mayor (alrededor de 3 ppm) en aquellas terminadas con una dieta a base de concentrado que en las terminadas a pasto. En cambio, en novillos no observaron efecto de la dieta. Por el contrario, Lanari et al. (2002) hallaron que tanto los animales terminados sobre pasturas como con dietas ricas en granos presentaron similar concentración de mioglobina. Una explicación a esta discrepancia fue sugerida por Gatellier et al. (2005), quienes señalan que el efecto confuso de la dieta sobre la concentración de mioglobina puede deberse a que ésta se incrementa con la edad (Renerre, 1990) o, a veces, no se conoce la historia previa de alimentación o si hubo suplementación con hierro de los animales. Es por esto que, cuando se comparan animales provenientes de pasturas o terminados con granos, establecer las diferencias en el color de la carne atribuidas a la dieta puede resultar confuso. Generalmente, los animales terminados sobre pasturas tienen más edad o menos grasa intramuscular, lo que no favorece a los parámetros colorimétricos (Dunne et al., 2011). En el trabajo de Lanari et al. (2002) los animales fueron faenados a la misma edad y en Bidner et al. (1986) presentaron una diferencia de 10 meses, con el propósito de que los animales engordados en pasturas presentaran un peso promedio similar al de los animales alimentados con grano.

Si bien la dieta no afecta directamente el estado de la mioglobina, podría hacerlo a través del aporte de antioxidantes. Estos pueden ser naturales o sintéticos, aunque la potencial toxicidad de los sintéticos ha creado por parte de los consumidores una mayor demanda hacia los antioxidantes naturales (Falowo et al., 2014). Estudios recientes han propuesto la utilización de compuestos antioxidantes naturales derivados de los alimentos, tales como vitamina E, carnosina, extractos de semillas de uva y catequinas de té. La vitamina E, actúa como antioxidante a nivel de la síntesis del pigmento hemo, está presente en las pasturas y se acumula en el tejido adiposo de los animales (Descalzo y Sancho, 2008; Pouzo et al., 2016). Estos antioxidantes naturales retrasan la formación de metamioglobina y evitan la decoloración temprana de la superficie de la carne expuesta al oxígeno (Liu et al., 1995; Faustman et al., 1998; O’Sullivan et al., 2003; Albertí et al., 2005).

Durante la terminación los animales acumulan grasa intramuscular y aumentan el peso corporal, lo cual se encuentra asociado a la hipertrofia de fibras rojas, aumento en la concentración de mioglobina y el consecuente aumento del parámetro $\mathrm{a}^{*}$ (redness), (Wood et al., 1999; Ozawa et al., 2000; Shibata et al., 2014; Kim et al., 2010; Jeong et al., 2012; Mialon et al., 2015). En cuanto a la concentración energética de las dietas, Johnston et al. (1981) y Joo et al. (2013) confirmaron que si bien el tipo de fibras en los distintos músculos está asociado con la raza de los animales, también la dieta tiene efecto. Estos autores demostraron que hay mayor porcentaje de fibras glucolíticas en dietas de feedlot que en die- 


\begin{tabular}{|c|c|c|c|c|c|}
\hline \multirow[b]{2}{*}{ Dieta } & \multicolumn{2}{|c|}{ Consumo } & \multicolumn{2}{|c|}{ Glucógeno* } & \multirow{2}{*}{$\begin{array}{c}\text { Animal/ } \\
\text { Duración/ } \\
\text { Referencia }\end{array}$} \\
\hline & kg MS/día & MJ EM/kg $g^{0,75}$ & $\begin{array}{c}\text { Final } \\
(\mu \mathrm{mol} / \mathrm{g})\end{array}$ & $\begin{array}{l}\text { Cambio } \\
(\mu \mathrm{mol} / \mathrm{g})\end{array}$ & \\
\hline Ensilaje & 5,9 & 0,81 & $\begin{array}{l}\text { SM: } 99^{B} \\
\text { ST: } 74\end{array}$ & $\begin{array}{c}\text { SM: } 16^{B} \\
\text { ST: } 2^{b}\end{array}$ & \multirow{2}{*}{$\begin{array}{l}\text { Novillos } \\
12 \text { meses } \\
(304 \mathrm{~kg})\end{array}$} \\
\hline Heno & 7,1 & 1,06 & $\begin{array}{l}\text { SM: } 106^{B} \\
\text { ST: } 72\end{array}$ & $\begin{array}{l}\text { SM: } 18^{B} \\
\text { ST: } 13^{b}\end{array}$ & \\
\hline Ensilaje + grano cebada & 7,2 & 1,08 & $\begin{array}{l}\text { SM: } 114^{A} \\
\text { ST: } 82\end{array}$ & $\begin{array}{l}\text { SM: } 35^{A} \\
\text { ST: } 29^{a}\end{array}$ & \multirow{2}{*}{$\begin{array}{l}\text { Tudor et al. } \\
\quad(1996)\end{array}$} \\
\hline Heno + grano de cebada & 6,3 & - & $\begin{array}{c}\text { SM:118 } \\
\text { ST: } 84\end{array}$ & $\begin{array}{l}\text { SM: } 33^{A} \\
\text { ST: } 28^{a}\end{array}$ & \\
\hline Ensilaje de gramíneas & 6,4 & 0,64 & $\mathrm{~L}: 8^{\mathrm{b}}$ & L: -8 & $\begin{array}{c}\text { Toros } \\
(480 \mathrm{~kg})\end{array}$ \\
\hline $\begin{array}{l}\text { Ensilaje de gramíneas }+7 \mathrm{~kg} \\
\text { concentrado energético }\end{array}$ & 9,5 & 1,19 & $L: 93^{a}$ & $\mathrm{~L}: 2$ & $\begin{array}{c}15 \text { días } \\
\text { Immonen et al. } \\
(2000)\end{array}$ \\
\hline Heno de pasturas & 6,98 & 0,72 & $\begin{array}{l}\text { SM: } 85^{b} \\
\text { ST: } 77\end{array}$ & - & \multirow{2}{*}{$\begin{array}{c}\text { Novillos } \\
18 \text { meses } \\
(330 \mathrm{~kg})\end{array}$} \\
\hline Ensilaje de pasturas & 7,81 & 1,02 & $\begin{array}{l}\text { SM: } 107^{\mathrm{a}} \\
\text { ST: } 88\end{array}$ & - & \\
\hline Grano de maíz & 11,77 & 1,70 & $\begin{array}{l}\text { SM: } 105^{a} \\
\text { ST: } 98\end{array}$ & - & \multirow{2}{*}{$\begin{array}{c}42 \text { días } \\
\text { Gardner et al. } \\
\text { (2001) }\end{array}$} \\
\hline Grano de cebada & 11,3 & 1,57 & $\begin{array}{l}\text { SM: } 113^{a} \\
\text { ST: } 94\end{array}$ & - & \\
\hline
\end{tabular}

tas de base pastoril y suplementación con granos. En ovinos, Solomon y Lynch (1988) estudiaron el tipo de fibras predominantes en la composición del músculo Longissimus, comparando dos dietas de diferente energía (7,8 vs. 11,7 MJ EM/kg MS). Los resultados obtenidos mostraron que hubo un predominio de fibras oxidativas cuando la dieta tuvo menor concentración energética, mientras que las fibras glucolíticas predominaron en los corderos alimentados con la dieta de mayor energía.

\section{GLUCÓGENO Y GRASA SUBCUTÁNEA \\ GeNERALIDADES}

El principal factor para asegurar la producción de carne de buena calidad, referida al color y la terneza, es mantener o lograr un descenso conjunto del $\mathrm{pH}$ y de la temperatura en las canales (Thompson, 2002; Lucero Borja et al., 2014). Para lograr este objetivo es necesario un adecuado nivel de glucógeno y de grasa subcutánea, variables que pueden ser manejadas a través de la dieta.

En el tracto digestivo de los rumiantes, los componentes dietarios son transformados en ácidos grasos volátiles, principalmente acético, propiónico y butírico, cuyas concentraciones dependen de la composición de la dieta. Estos ácidos son metabolizados por los microorganismos ruminales, o se absorben para ser utilizados por el animal (Orskov y Ryle, 1990; Fran- ce y Dijkstra, 2005). Los bovinos absorben muy poca cantidad de carbohidratos dietarios, incluso cuando su alimentación es con granos ricos en almidón no degradable en el rumen. Se calcula que la absorción de glucosa en el intestino puede representar sólo el 30\% del total de la glucosa utilizada por el animal (Brockman, 1993). Para suplir la baja absorción, la glucosa es neosintetizada en el hígado a partir de precursores gluconeogénicos. Se estima que del $90 \%$ de la glucosa neosintetizada, aproximadamente el $73 \%$ proviene del ácido propiónico, el 13\% del ácido láctico, el 12\% de los aminoácidos potencialmente glucogénicos (glicina y serina) y lo restante del glicerol (Lindsay, 1993). La inclusión de maíz en la dieta incrementa la producción de ácido propiónico, aumentando la gluconeogénesis, que es sustrato dependiente y, por lo tanto, la disponibilidad de glucosa en la sangre (Lindsay, 1993). De la glucosa sanguínea transportada, el músculo sólo almacena el 3\% como glucógeno (Brockman, 1993).

Si la dieta contiene lípidos, los ácidos grasos de los triglicéridos pueden utilizarse directamente como fuente de energía ( $\beta$-oxidación), o almacenarse en el tejido adiposo. La vía metabólica que sigan dependerá de la regulación hormonal establecida para mantener la homeostasis energética (Pethick et al., 2004). Sólo el glicerol puede ser utilizado en la gluconeogénesis (Gurr et al, 2002; Pethick et al., 2004; Hess et al., 2008). Cuando las dietas incluyen fuentes lipídicas como su- 
plemento concentrado en energía, se ha observado que los animales en crecimiento tienden a incrementar los depósitos de grasa corporal a partir de los ácidos grasos provenientes directamente de la dieta (Andrae et al., 2001). Asimismo, un incremento de ácidos grasos en plasma podría disminuir el catabolismo de la glucosa a través de la secreción de insulina, generando una mayor capacidad de almacenamiento de glucosa en el tejido muscular y hepático (Chilliard, 1993).

\section{EFECTO DE LA DIETA}

En general, cuando la dieta es pastoril y los animales se suplementan con concentrados energéticos, se han observado mejoras en la respuesta productiva y en la acumulación de grasa subcutánea (Pavan et al., 2007; Dawson et al, 2010; Scholljegerdes y Kronberg, 2008; Pouzo et al., 2015). Son escasos los trabajos que evaluaron el efecto de la dieta sobre la concentración del glucógeno almacenado en el músculo, que es el factor que más se relaciona con el $\mathrm{pH}$; este último afecta el color y la terneza de la carne como se comentó anteriormente. Si bien el efecto de la dieta sobre el glucógeno muscular no está del todo claro, se ha evaluado la importancia tanto de la energía contenida en la dieta (Immonen et al., 2000) como de la ingesta total de energía metabolizable (Gardner et al., 2014) sobre los niveles de glucógeno y el pH de la carne. En base a estas dos posturas se resumió la información mostrada en la tabla I. Como se puede observar, los trabajos publicados son poco concluyentes y no se observan resultados que apoyen a alguna de las dos posturas. Lo que se puede observar es que las dietas que contenían grano incrementaron la concentración del glucógeno, entre 2 y $35 \mu \mathrm{mol} / \mathrm{g}$, en los músculos con metabolismo óxido glicolítico (Longissimus y Semimembranosus) y no afectaron de la misma manera al músculo Semitendinosus, cuyo metabolismo es glicolítico. De acuerdo a Immonen et al. (2000) el incremento de glucógeno con la dieta rica en energía implicó menor $\mathrm{pH}$ (0,65 puntos), mientras que Tudor et al. (1996) y Gardner et al. (2001) no indicaron los valores de $\mathrm{pH}$. Si bien hace falta más información al respecto, una forma de poder comparar los resultados de distintos ensayos es expresando el consumo de energía metabolizable por unidad de peso metabólico. De esta manera se podría establecer que el incremento en la concentración de glucógeno ocurre cuando el consumo de energía metabolizable se encuentra por encima de 1,0-1,1 MJ EM/ $/ \mathrm{kg}^{0,75}$.

En la medida que aumenta el peso vivo de los animales, mayor es la energía consumida que se retiene en forma de grasa, por lo que la ganancia diaria de peso disminuye (NRC, 2000; Jurie et al., 2005). El aumento de la deposición de grasa se evidencia en el engrasamiento general de la carcasa, el incremento del espesor de grasa dorsal y el mayor contenido de grasa intramuscular (Wood et al., 2008). Cuando los animales consumen ad libitum dietas ricas en concentrados energéticos, tienden a producir carcasas con mayor cobertura grasa, en comparación a otros que consumen dietas fibrosas durante el mismo período de tiempo. French et al. (2001) evaluaron la respuesta productiva de animales con seis dietas diferentes, encontrando que las dietas más energéticas, es decir las que contenían granos, incrementaron la deposición de grasa en distintos lugares de las carcasas (tabla II). En el mismo sentido, Cerdeño et al. (2006) observaron mayor cantidad de grasa visceral (renal, retroperitoneal, omental y mesentérica) en animales que consumieron concentrado ad libitum y disminución gradual de este depósito en los que consumieron concentrado en forma más restringida. Los resultados obtenidos se deberían a la concentración de energía en cada estrategia de alimentación y, por tanto, al promedio de ganancia diaria de peso en cada grupo.

\section{Cambios en la estructura del colágeno \\ GENERALIDADES}

El tejido conectivo está constituido principalmente por fibras proteicas de colágeno y elastina, rodeadas por una matriz de proteoglicanos. En la materia seca de la carne el contenido total de colágeno en distintos músculos del bovino varía de 1 a $15 \%$, en tanto que la elastina se presenta en menor proporción, de 0,6 a 3,7\% (Bendall, 1967).

El colágeno se compone de subunidades denominadas tropocolágeno que tienen un alto contenido de aminoácidos, entre ellos glicina, prolina e hidroxiprolina. Las moléculas de tropocolágeno están alineadas en paralelo y se mantienen unidas por enlaces intermoleculares covalentes cruzados que le brindan termoestabilidad. Estos enlaces se incrementan a medida que avanza la edad de los animales, disminuyendo la solubilidad del colágeno (Etherington y Sims, 1981).

Tabla II. Tipo de dietas y su efecto sobre la deposición de grasa (Diet types and effect on fat increase).

\begin{tabular}{|c|c|c|c|c|c|c|c|}
\hline & \multicolumn{6}{|c|}{ Tratamientos dietarios } & \multirow[t]{2}{*}{ Error estándar } \\
\hline & C1 & $\mathrm{C} 2$ & C3 & $\mathrm{C} 4$ & C5 & C6 & \\
\hline Engrasamiento canal ${ }^{1}$ & $3,73^{a, b}$ & $4,00^{\mathrm{a}}$ & $4,13^{a}$ & $3,93^{a}$ & $3,13^{b}$ & $4,20^{\mathrm{a}}$ & 0,22 \\
\hline Grasa pélvico renal, kg & $5,21^{c}$ & $8,15^{b}$ & $9,64^{\mathrm{a}, \mathrm{b}}$ & $8,63^{a, b}$ & $3,31^{c}$ & $10,49^{a}$ & 0,69 \\
\hline Grasa pélvico renal, g/kg canal & $17^{\mathrm{b}, \mathrm{c}}$ & $25^{a, b}$ & $28^{a}$ & $27^{a}$ & $12^{c}$ & $31^{\mathrm{a}}$ & 3,1 \\
\hline
\end{tabular}

C1= asignación $11 \mathrm{~kg}$ de forraje/an./día; C2= C1 más 2,5 kg/an./día de concentrado energético; C3= C1 más 5 kg/an./día de concentrado energético; C4= asignación 22 kg de forraje/an./día; C5= pastoreo sobre forraje rechazado en C4; C6= concentrados ad libitum más 1 kg/ an./día de fibra.

${ }^{1}$ Engrasamiento canal: Valoración del grado de engrasamiento según la clasificación utilizada en la Unión Europea (1= más magra; 5= más engrasada).

Letras distintas indican diferencias significativas $(p<0,05)$ dentro de una misma fila.

Adaptado de French et al. (2001). 


\section{EFECTO DE LA DIETA}

Los distintos estudios indican que la dieta modifica el contenido de colágeno total o soluble al afectar la tasa de aumento de peso diario. Las dietas ricas en energía, en comparación con las pastoriles, o la suplementación energética en dietas basadas en pasturas, permiten mayor aumento diario de peso (Chicatún et al., 2006, García et al., 2008; Latimori et al., 2008). Al aumentar la tasa de ganancia se genera un efecto de dilución del colágeno debido a la hipertrofia de las miofibrillas, lo que resulta en una disminución del colágeno total (Archile Contreras et al., 2010). Por otro lado, esas dietas energéticas aumentan la proporción de colágeno soluble (Miller et al., 1987; Cranwell et al., 1996; Silva et al., 2010), lo cual se podría deber a que la mayor tasa de crecimiento va acompañada por un incremento en el catabolismo y síntesis de nuevas fibras de colágeno, que están menos polimerizadas (McCormick, 1994; Sylvestre et al., 2002). Miller et al. (1983) sugirieron que el mecanismo por el cual se modifican las concentraciones de colágeno en animales jóvenes se debe a que, en la etapa de crecimiento muscular rápido, la dieta energética demora la síntesis y maduración del colágeno, lo que resulta en una mayor fracción soluble (Miller et al., 1987; Therkildsen et al., 2008).

Schnell et al. (1997) indicaron que el efecto de una dieta energética depende también del tiempo de su- ministro, además de lo que se mencionó en párrafos anteriores. Al comparar 0, 14, 28, 42 y 56 días de suplementación con una misma dieta, observaron que el contenido de colágeno soluble en el músculo Longissimus fue aumentando hasta el día 28, a partir del cual se estabilizó. Este trabajo (Schnell et al., 1997) fue el único que evaluó el efecto del tiempo de suministro de una dieta sobre el colágeno muscular, lo que sugiere la necesidad de realizar más trabajos para evaluar el efecto de la duración de los tratamientos dietarios. Sin embargo, es poco probable que se hagan ensayos de muy corta duración, ya que para estudiar el efecto de una dieta la evaluación en general supera los 28 días.

El efecto del tipo de dieta y el tiempo de suministro de una misma dieta sobre la composición del colágeno se resumen en la tabla III.

\section{ACTIVIDAD DE LAS ENZIMAS PROTEOLÍTICAS \\ Generalidades}

La maduración es el proceso mediante el cual la carne aumenta la terneza cuando es almacenada determinado tiempo a cierta temperatura (Koohmaraie,1996). Durante dicho proceso ocurre la proteólisis miofibrilar, lo que mejora la terneza de la carne sin que ocurran cambios en la longitud de los sarcómeros (Koohmaraie,1996). En esta etapa hay fragmentación

Tabla III. Efecto de la dieta sobre la concentración del colágeno (Diet effect on collagen concentration).

\begin{tabular}{|c|c|c|c|c|}
\hline \multirow[t]{2}{*}{ Tratamiento/Dieta } & \multirow{2}{*}{$\begin{array}{c}\text { Aumento diario } \\
\text { peso, } \mathrm{kg} / \mathrm{d} \text { ía }\end{array}$} & \multicolumn{2}{|c|}{ Concentración de colágeno } & \multirow[t]{2}{*}{ Animales/ Duración / Referencias } \\
\hline & & Total & Insoluble & \\
\hline & & & & Vacas de 10 años \\
\hline Alta energía & 1,00 & a & a & 75 días \\
\hline Baja energía & 0,10 & $\mathrm{~b}$ & $\mathrm{~b}$ & Miller et al. (1987) \\
\hline Grano (85\%) & - & ns & ns & $\begin{array}{l}\text { Novillos faenados con } 1 \mathrm{~cm} \text { de espesor de } \\
\text { grasa dorsal }\end{array}$ \\
\hline Forraje $(100 \%)$ & - & ns & ns & Cox et al. (2006) \\
\hline Heno + alto concentrado & 1,34 & ns & ns & Terneros de 6 meses \\
\hline Heno + bajo concentrado & 1,23 & ns & ns & 4 meses \\
\hline Forraje + alto concentrado & 1,36 & ns & ns & \\
\hline Forraje + bajo concentrado & 1,26 & ns & ns & Serrano et al. (2007) \\
\hline Concentrado (85\%) & 2,04 & $\mathrm{~b}$ & a & Novillos de 1 año \\
\hline Forraje de alfalfa & 1,33 & a & $\mathrm{b}$ & 108 días \\
\hline Forraje de gramínea & 1,31 & a & $a b$ & Archile Contreras et al. (2010) \\
\hline Concentrados ad libitum & 1,49 & ns & - & Novillos 162 kg \\
\hline $\begin{array}{l}\text { Concentrado + semilla de soja (ad li- } \\
\text { bitum) }\end{array}$ & 1,48 & ns & - & 148 días \\
\hline $\begin{array}{l}\text { Concentrado + semilla de soja (limita- } \\
\text { dos a } 0,6 \mathrm{~kg} / \mathrm{bocado} \text { ) }\end{array}$ & 1,37 & ns & - & Marti et al. (2014) \\
\hline \multicolumn{5}{|l|}{ Días de suplementación } \\
\hline 0 & 0 & $\mathrm{~b}$ & ns & Vacas de 4 a 10 años \\
\hline 14 & $-0,05$ & $a b$ & ns & \\
\hline 28 & 1,04 & a & ns & \\
\hline 42 & 1,30 & a & ns & \\
\hline 56 & 1,74 & a & ns & Schnell et al. (1997) \\
\hline
\end{tabular}

a,b: diferencias significativas entre dietas ns: diferencias no significativas. 
de los miofilamentos producida por enzimas proteolíticas intracelulares e intralisosómicas. La m-calpaína y la $\mu$-calpaína son proteasas intracelulares calciodependientes que difieren en su sensibilidad ante la presencia de estos iones, y su actividad está regulada por su inhibidor natural, la calpastatina (Soria y Corva 2004; Geesink et al., 2006). Asimismo, existen también las catepsinas, que son proteasas que al romperse las membranas lisosomales luego de la caída del pH, se liberan al citoplasma y a los espacios intercelulares (Cheret et al., 2007). Todas estas enzimas actúan durante la proteólisis post mortem mejorando la terneza.

\section{EFECTO DE LA DIETA}

Como se explicó en otras secciones, las dietas de alta energía permiten el mayor crecimiento de los animales en un corto período de tiempo. Incrementar la tasa de crecimiento genera cambios en la dinámica de intercambio proteico, donde se establece un nuevo equilibrio entre el anabolismo y catabolismo (Dransfield y Sosnicki, 1999). La hipertrofia muscular puede darse por un aumento en la síntesis proteica o por una disminución en el catabolismo. Esta última parece ser la vía más común, como ha sido bien documentado a través del estudio de ovinos con la mutación callipyge, o a través del suministro de agonistas $\beta$ adrenérgicos (Goll et al., 1998; Koohmaraie et al., 2002). Para que se produzca la hipertrofia muscular, debe haber un aumento en la actividad supresora de la calpastatina sobre la m-calpaína y u-calpaína, con la consecuente reducción del recambio proteico a nivel miofibrilar (Goll et al., 1998; Kirchofer et al., 2002; Veiseth-Kent et al., 2010).

El tipo de fibra muscular y/o el metabolismo inducido en ellas a partir de la dieta, tiene un rol importante en la actividad de las enzimas proteolíticas. El consumo de dietas de baja energía estimula la hipertrofia de las fibras rojas (Moody et al., 1980; Johnston et al., 1981). Por otra parte, Ouali y Talmant (1990) y Koohmaraie (1996) indicaron que la concentración de las enzimas proteolíticas disminuye en los músculos de contracción rápida y metabolismo glucolítico. En este mismo sentido, Du Toit (2011) indicó que la concentración de ácido láctico muscular (menor $\mathrm{pH}$ ) presenta una asociación negativa con la actividad de la $\mu$-calpaína.

Una hipótesis sobre la cual se está trabajando actualmente indica que las dietas que permiten la deposición de ácidos grasos poliinsaturados favorecerían la actividad de las enzimas proteolíticas. De acuerdo a lo estudiado por Chao et al. (2015), el incremento de los ácidos grasos poliinsaturados en las membranas del retículo sarcoplasmático disminuiría su estabilidad post mortem. Lo anterior se debería a que los fosfolípidos en la membrana del retículo sarcoplasmático se degradan durante la maduración post mortem y que el calcio se filtra a través de los canales formados por esta degradación en la membrana. Es probable que la diferencia en el perfil de ácidos grasos del retículo no sea el único factor que contribuya a las diferencias en la terneza y la concentración de calcio libre (Chao et al., 2015). Wilkerson (2016), siguiendo con estos estudios observó que un mayor contenido de ácidos grasos poliinsaturados depositados en las mitocondrias, usadas como modelo de retículo sarcoplasmático, afecta el flujo de calcio por el aumento de la susceptibilidad a la oxidación de los lípidos de membrana. Por lo tanto, el efecto de los ácidos grasos poliinsaturados sobre la terneza de la carne puede ser inhibido con la suplementación con vitamina E.

Mediante la suplementación de los animales con fuentes de calcio es otra manera de afectar la terneza de la carne. Los resultados obtenidos por Swanek et al. (1999) y Montgomery et al. (2002) indicaron que los novillos suplementados con vitamina D presentaron mayor concentración de calcio en plasma y músculo Longissimus, en comparación a los animales no suplementados. Los aumentos en el contenido de calcio se vieron reflejados en el aumento de la terneza, atribuible a la activación de las calpaínas (Swanek et al., 1999) o aumento en la longitud de los sarcómeros (Rafalska, 2016). Los sarcomeros más largos permiten mayor contacto de las enzimas proteolíticas con las proteínas miofibrilares (Weaver et al., 2008).

\section{CONCLUSIONES}

Las dietas de baja energía, como ser aquellas basadas en forrajes, son usualmente relacionadas con carnes más oscuras y menos tiernas. Esto se debe a que los animales con este tipo de dietas tienen un bajo depósito de glucógeno muscular, lo que puede disminuir la velocidad del descenso del $\mathrm{pH}$ (no favorable en reses que se enfrían rápidamente), o no alcanzar para que el pH final sea suficientemente bajo. En consecuencia, a mayor pH la carne presenta color más oscuro.

Por otro lado, las dietas fibrosas estimulan el metabolismo oxidativo de las fibras musculares, lo cual es acompañado por el incremento de la concentración de mioglobina. Sin embargo, en este punto se pueden presentar efectos confundidos de la dieta y la edad sobre la calidad de la carne, dado que los animales en pastoreo tienen menor ganancia de peso en comparación con los alimentados con concentrados energéticos. La menor ganancia de peso implica mayor edad al momento de faena, o faena de animales no terminados donde el espesor de grasa dorsal es inferior al recomendado en la bibliografía. En el caso de que la grasa dorsal no sea suficiente para amortiguar el descenso de temperatura de la res, se genera un desajuste en la relación entre la caída del pH y la temperatura post mortem, lo que inevitablemente provocará cambios indeseables en el color. Por otra parte, estos dos factores afectan a la longitud de los sarcómeros, la cual está asociada positivamente con la terneza.

La tasa de ganancia de peso también influye en la composición del colágeno, siendo menor la fracción soluble cuando los animales son alimentados con dietas que implican un lento crecimiento. Para que haya ganancia de peso debe haber hipertrofia muscular mediante la supresión de las enzimas proteolíticas. Estas enzimas son las que producirán la proteólisis de las miofibras post mortem facilitando la tenderización.

Por todo esto, desde el punto de vista de la nutrición animal, es necesario mantener un adecuado nivel de alimentación y que las dietas aporten suficiente 
energía y proteína, lo que asegurará, de manera directa o indirecta, la obtención de carnes con atributos sensoriales coincidentes con los requeridos por un gran número de consumidores.

\section{AGRADECIMIENTOS}

El trabajo implicado fue financiado por FONCYT (Fondo para la Investigación Científica y Tecnológica), CONICET (Consejo Nacional de Investigaciones Científicas y Técnicas), la Universidad Nacional de Mar del Plata y la Estación Experimental Agropecuaria Balcarce (INTA), de la República Argentina.

\section{BIBLIOGRAFÍA}

Albertí, P.; Panea, B.; Ripio, G.; Sañudo, C.; Olleta, J.L.; Heguerela, I.; Campo, M.M. y Serra, X. 2005. Medición del color. En: Cañeque, V.y Sañudo, C. (Eds.). Estandarización de las metodologías para evaluar la calidad del producto (animal vivo, canal, carne y grasa) en los rumiantes. Instituto Nacional de investigación y Tecnología Agraria y Alimentaria. Madrid. España. pp. 216-225.

Andrae, J.G.; Duckett, S.K.; Hunt, C.W.; Pritchard, G.T. and Owens, F.N. 2001. Effects of feeding high-oil corn to beef steers on carcass characteristics and meat quality. J Anim Sci, 79: 582-588.

Archile-Contreras, A.C.; Mandell, I.B. and Purslow, P.P. 2010. Disparity of dietary effects on collagen characteristics and toughness between two beef muscles. Meat Sci, 86: 491-497.

Bendall, J.R. 1967. The elastin content of various muscles of beef animals. J Sci Food Agric, 18: 553-558.

Bidner, T.D.; Schupp, A.R.; Mohamad, A.B.; Rumore, N.C.; Montgomery, R.E.; Bagley C.P. and Mcmillin, K.W. 1986. Acceptability of beef from Angus-Hereford or Angus-Hereford-Brahman steers finished on allforage or a high-energy diet. J Anim Sci, 62: 381-387.

Brockman, R.P. 1993. Glucose and short chain fatty acid metabolism. In: Forbes, J.M. and France, J. (Eds). Quantitative aspects of ruminant digestion and metabolism. CAB International. UK. pp: 249-265.

Cerdeño, A.; Vieira, C.; Serrano, E.; Lavın P. and Mantecón, A.R. 2006. Effects of feeding strategy during a short finishing period on performance, carcass and meat quality in previously-grazed young bulls. Meat Sci, 72: 719-726.

Chao, M.D.; Domenech, K.I. and Calkins, C.R. 2015. A basic mechanism of beef tenderization: feeding wet distillers grains plus solubles contributes to sarcoplasmic reticulum membrane instability. Nebraska Beef Cattle Reports. Paper 847.

Chao, M.D.; Domenech K.I. and Calkins, C.R. 2016. Feeding vitamin E may reverse sarcoplasmic reticulum membrane instability caused by feeding wet distillers grains plus solubles to cattle. Nebraska Beef Cattle Reports. Paper 867.

Cheret, R.; Delbarreladrat, C.; Lamballerieanton M. and Verrezbagnis, V. 2007. Calpain and cathepsin activities in post mortem fish and meat muscles. Food Chemist, 101: 1474-1479.

Chicatún, A.; Santini, F.J.; Depetris, G.J.; Faverín C. y Villarreal, E. 2006. Calidad de la carne de novillos producidos bajo distintas estrategias de suplementación. Rev Arg Prod Anim, 26 (Sup. 1): 409-410.

Chilliard, Y. 1993. Dietary fat and adipose tissue metabolism in ruminants, pigs, and rodents: a review. J Dairy Sci, 76: 3897-3931.

Cox, R.B.; Kerth, C.R.; Gentry, J.G.; Prevatt, J.W.; Braden K.W. and Jones W.R. 2006. Determining acceptance of domestic forage- or grain-finished beef by consumers from three southeastern US states. J Food Sci, 71: 542-546.

Cranwell, C.D.; Unruh, J.A.; Brethour J.R. and Simms D.D. 1996. Influence of steroid implants and concentrate feeding on carcass and Longissimus muscle sensory and collagen characteristics of cull beef cows. J Anim Sci, 74: 1777-1783.

Dawson, A.M.; Fearonb, L.E.R.; Mossb, B.W. and Woods, V.B. 2010. Effects of substitution of a proportion of the concentrate in grass silage/ concentrate-based diets with extruded linseed on performance and meat quality of dairy bulls. Anim Feed Sci Tech, 156: 10-18.

Descalzo, A.M. and Sancho, A.M. 2008. A review of natural antioxidants and their effects on oxidative status, odour and quality of fresh beef produced in Argentina. Meat Sci, 79: 423-436.

Dransfield, E. and Sosnicki, A.A. 1999. Relationship between muscle growth and poultry meat quality. Poultry Sci, 78: 743-746.

Du Toit, E. 2011. Effects of production systems on the muscle status post mortem and meat quality of beef cattle. M. Sc. Thesis. University of Pretoria.

Dunne, P.G.; Monahan F.J. and Moloney, A.P. 2011 . Current perspectives on the darker beef often reported from extensively-managed cattle: Does physical activity play a significant role?. Livest Sci, 142: 1-22.

Etherington, D.J. and Sims, T.J. 1981. Detection and estimation of collagen. J Sci Food Agric, 32: 539-546.

Falowo, A.B.; Fayemi, P.O. and Muchenje, V. 2014. Natural antioxidants against lipid-protein oxidative deterioration in meat and meat products: A review. Food Res Int, 64, 171-181.

Faustman, C.; Chan, W.K. M.; Schaefer D.M. and Havens A. 1998. Beef color update: The role for vitamin E. J Anim Sci, 76: 1019-1026.

France, J. and Dijkstra, J. 2005. Volatile fatty acid production. In: Dijkstra, J.; Forbes. J. M. and France, J. (Eds.). Quantitative aspects of ruminant digestion and metabolism. CABI International. UK. pp. 157-175.

French, P.; O'Riordan, E.G.; Moloney, A.P.; O'Kiely, P. and Caffrey, P.J. 2001. Effects of concentrate level and grazing system on the performance of beef cattle grazing autumn herbage. Irish J Agricul Food Research, 33-44.

García, P.T.; Pensel, N.; Sancho, M.; Latimori, N.J.; Kloster, M.; Amigone, M. and Casal, J.J. 2008. Beef lipids in relation to animal breed and nutrition in Argentina. Meat Sci., 79: 500-508.

Gardner, G.E.; Mcintyre, B.L.; Tudor, G.D. and Pethick, D.W. 2001. The impact of nutrition on bovine muscle glycogen metabolism following exercise. Austr J Agricult Res, 52: 461-470.

Gardner, G.E.; McGilchrist, P. and Pethick, D.W. 2014. Ruminant glycogen metabolism. Anim Prod Sci, 54: 1575-1583.

Gatellier, P.; Mercier, Y.; Juin, H. and Renerre, M. 2005. Effect of finishing mode (pasture or mixed diet) on lipid composition, colour stability and lipid oxidation in meat from Charolais cattle. Meat Sci, 69: 175-186.

Geesink, G.H.; Kuchay, S.; Chishti A.H. and Koohmaraie, M. 2006. $\mu$-Calpain is essential for post mortem proteolysis of muscle proteins. J Anim Sci, 84: 2834-2840.

Goll, D.E.; Thompson, V.F.; Taylor R.G. and Ouali, A. 1998. The calpain system and skeletal muscle growth. Can J Anim Sci, 78: 503-512.

Gurr, M.I.; Harwood, J.L. and Frayn, K.N. 2002. Lipid biochemistry. Blackwell Science. Oxford. pp. 199-212.

Hess, B.W.; Moss, G.E. and Rule, D.C. 2008. A decade of developments in the area of fat supplementation research with beef cattle and sheep. $J$ Anim Sci, 86:188-204.

Immonen, K.; Ruusunen, M.; Hissa, K. and Puolanne E. 2000. Bovine muscle glycogen concentration in relation to finishing diet, slaughter and ultimate $\mathrm{pH}$. Meat Sci, 55: 25-31.

Jacob, R.H.; Surridge, V.S.M.; Beatty, D.T.; Gardner G.E. and Warner. R.D. 2014. Grain feeding increases core body temperature of beef cattle. Anim Prod Sci, 54: 444-449.

Jeong, J.Y.; Kim, G.D.; Ha, D.M.; Park, M.J.; Park, B.C.; Joo, S.T. and Lee C.Y. 2012. Relationships of muscle fiber characteristics to dietary energy density, slaughter weight, and muscle quality traits in finishing pigs. J A Sci Technol, 54: 175-183.

Johnston, D.M.; Moody, W.G.; Boling, J.A. and Bradley N.W. 1981. Influence of breed type, sex, feeding systems and muscle bundle size on bovine fibre type characteristics. J Food Sci, 46: 1760.

Joo, S.T.; Kim, G.D.; Hwang Y.H. and Ryu, Y.C. 2013. Control of fresh meat quality through manipulation of muscle fiber characteristics. Meat Sci, 95: 828-836.

Jurie, C.; Martin, J.F.; Listrat, A.; Jailler, R.; Culioli, J. and Picard, B. 2005. Effects of age and breed of beef bulls on growth parameters, carcass and muscle characteristics. Anim Sci, 80: 257-263. 
Killinger, K.M.; Calkins, W.J.; Umberger W.J.; Feuz, D.M. and Eskridge K.M. 2004. Consumer visual preferences and value for beef steaks differing in marbling level and color. J Anim Sci, 82: 3288-3293.

Kim, G.; Jeong, J.; Hur, S.; Yang, H.; Jeon, J. and Joo, S. 2010. The relationship between meat color (CIE L and a), myoglobin content, and their influence on muscle fiber characteristics and pork quality. Korean J Food Sci Anim Resour, 30: 626-633.

Kirchofer, K.S.; Calkins C.R. and Gwartney, B.L. 2002. Fiber type composition of muscles of the beef chuck and round. J Anim Sci, 80: 2872-2878

Koohmaraie, M. 1996. Biochemical factors regulating the toughening and tenderisation process of meat. Meat Sci, 43: 193-201.

Koohmaraie, M.; Kent, M.P.; Shackelford, S.D.; Veiseth E. and. Wheeler, T.L. 2002. Meat tenderness and muscle growth: is there any relationship? Meat Sci, 62: 345-352.

Lanari, M.C.; Brewster, M.; Yang, A. and Tume, R.K. 2002. Pasture and grain finishing affect the color stability of beef. J Food Sci, 67: 2467-2473

Latimori, N.J.; Kloster, A.M.; García, P.T.; Carduza, F.J.; Grigioni, G. and Pensel, N.A. 2008. Dietand genotype effects on the quality index of beef produced in the Argentine Pampeana region. Meat Sci, 79: 463-469.

Lefaucheur, L. 2010. A second look into fibre typing. Relation to meat quality. Meat Sci, 84: 257-270.

Lindsay, D. B. 1993. Metabolism of the portal drained viscera. In: Forbes. J. M. and France, J. (Eds.). Quantitative aspects of ruminant digestion and metabolism. CAB International UK. pp. 267-298.

Listrat, A.; Lebret, B.; Louveau, I.; Astruc, T.; Bonnet, M.; Lefaucheur, L.; Picard, B. and Bugeon, J. 2016. How muscle structure and composition influence meat and flesh quality. Scientific World J, 2016. Article ID 3182746. http://dx.doi.org/10.1155/2016/3182746.

Liu, Q.; Lanari, M.C. and Schaefer, D.M. 1995. A review of dietary vitamin e supplementation for improvement of beef quality. J Anim Sci, 73: 3131-3140.

Lucero-Borja, J.; Pouzo, L.B.; De La Torre, M.S.; Langman, L.; Carduza, F.; Corva, P.M.; Santini, F.J. and Pavan, E. 2014. Slaughter weight, sex and age effects on beef shear force and tenderness. Livest Sci, 163: 140-149

Lusk, J.L.; Fox, J.A.; Schroeder, T.C.; Mintert J. and Koohmaraie, M. 2001. In store valuation of steak tenderness. Am J AgricultEcon, 83: 539-550.

Marti, S.; Pérez, M.; Aris, A.; Bach, A. and Devant, M. 2014. Effect of dietary energy density and meal size on growth performance, eating pattern, and carcass and meat quality in Holstein steers fed highconcentrate diets. J Anim Sci, 92: 3515-3525.

McCormick, R.J. 1994. The flexibility of the collagen compartment of muscle. Meat Sci, 36: 79-91.

Mialon, M.M.; Renand, G.; Ortigues-Marty, I.; Bauchart, D.; Hocquette, J.F.; Mounier, L.; Noël, T.; Micol, D. and Doreau. M. 2015. Fattening performance, metabolic indicators, and muscle composition of bulls fed fiber-rich versus starch plus lipid-rich concentrate diets. J Anim Sci, 93: 319-333.

Miller, M.F.; Cross, H.R.; Crouse, J.D. and Jenkins, T.G. 1987. Effect of feed energy intake on collagen characteristics and muscle quality of mature cows. Meat Sci, 21: 287-294.

Miller, R.K.; Tatum, J.D.; Cross, H.R.; Bowling, R.A. and Clayłon, R.P. 1983. Effects of carcass maturity on collagen solubility and palatability of beef from grain finished steers. J Food Sci, 48: 484-486.

Montgomery, J.L.; Carr, M.A.; Kerth, C.R.; Hilton, G.G.; Price, B.P.; Galyean, M.L.; Horts, R.L.; Miller, M.F. 2002. Effect of vitamin D3 supplementation level on the postmortem tenderization of beef from steers. J Anim Sci, 80: 971-981.

Moody, W.G.; Kemp, J.D.; Mahyuddin, M.; Johnston, D.M. and Ely, D.G. 1980. Effect of feeding systems slaughter weight and sex on histological properties of lamb carcasses. J Anim Sci, 50: 249-256.

Muir, P.D.; Deaker, J.M. and Bown, M.D. 1998. Effects of forage and grain-based feeding systems on beef quality: A review. N Z J Agric Res, 41:623-635.
NRC. 2000. Nutrient Requirements of Beef Cattle. National Academy Press. Washington, DC. pp. 3-16.

O'Sullivan, A.; Galvin, K.; Moloney, A.P.; Troy, D.J.; O’Sullivan, K. and Kerry, J.P. 2003. Effect of pre-slaughter rations of forage and/or concentrates on the composition and quality of retail packaged beef. Meat Sci, 63: 279-286.

Orskov, E.R. and Ryle, M. 1990. Energy nutrition in ruminants. Elsevier Applied Science. London. 149 pp.

Ouali, A., and Talmant, A. 1990. Calpains and calpastatin distribution in bovine, porcine and ovine skeletal muscles. Meat Sci, 28: 331-348.

Ozawa, S.; Mitsuhashi, T.;Mitsumoto, M.; Matsumoto, S.; Itoh, N.; Itagaki, K.; Kohnof, T. and Dohgo. T. 2000. The characteristics of muscle fiber types of Longissimus thoracis muscle and their influences on the quantity and quality of meat from Japanese Black steers. Meat Sci, 54: 65-70.

Pavan, E.; Duckett, S.K. and Andrae, J.G. 2007. Corn oil supplementation to steers grazing endophyte-free tall fescue. I. Effects on in vivo digestibility, performance, and carcass traits. J Anim Sci, 85: 1330-1339.

Pethick, D.W.; Harper, G.S. and Oddy, V.H. 2004. Growth, development and nutritional manipulation of marbling in cattle: a review. Anim Prod Sci, 44: 705-715.

Popp, J.; Wicke, M.; Klein, G. and Krischek, C. 2015. The relationship of pork Longissimus muscle $\mathrm{pH}$ to mitochondrial respiratory activities, meat quality and muscle structure. Anim, 9: 356-361.

Pouzo, L.B.; Descalzo, A.; Zaritzky, N.E.; Rossetti, L. and Pavan, E. 2016. Antioxidant status, lipid and color stability of aged beef from grazing steers supplemented with corn grain and increasing levels of flaxseed. Meat Sci, 111: 1-8.

Pouzo, L.B.; Fanego, N.; Santini, F.J.; Descalzo, A. and Pavan, E. 2015. Animal performance, carcass characteristics and beef fatty acid profile of grazing steers supplemented with corn grain and increasing amounts of flaxseed at two animal weights during finishing. Livest Sci, 178: 140-149.

Priolo, A.; Micol, D. and Gabriel, A.J. 2001. Effects of grass feeding systems on ruminant meat colour and flavour. Review. Anim Res, 50: 185-200.

Purslow, P.P. 2005. Intramuscular connective tissue and its role in meat quality. Review. Meat Sci, 70: 435-447.

Rafalska, U.K. 2016. Influence of dietary vitamin D 3 supplementation on the sarcomere length, Warner Bratzler shear force, shortening of ageing time, and sensory acceptance of culinary beef muscles. Turkish J Vet Anim Sci, 40: 514-520.

Renerre, M.T. 1990. Factors involved in the discoloration of beef meat. Interl J Food Sci Tech, 25: 613-630.

Resurrección, A.V.A. 2004. Sensory aspects of consumer choices for meat and meat products. Meat Sci, 66: 11-20.

Ryu, Y.C. and Kim, B.C. 2005. The relationship between muscle fiber characteristics, post mortem metabolic rate, and meat quality of pig Longissimus dorsi muscle. Meat Sci, 71: 351-357.

Schnell, T.D.; Belk, K.E.; Tatum, J.D.; Miller R.K. and Smith, G.C. 1997. Performance, carcass, and palatability traits for cull cows fed highenergy concentrate diets for $0,14,28,42$, or 56 days. J Anim Sci, 75 : 1195-1202.

Scholljegerdes, E.J. and Kronberg, S.L. 2008. Influence of level of supplemental whole flaxseed on forage intake and site and extent of digestion in beef heifers consuming native grass hay. J Anim Sci, 86: 2310-2320.

Seideman, C.; Smithl, C. and Durlandj, P.R. 1984. Factors associated with fresh meat color: heme-chemistry. Meat Sci, 6: 211-237.

Serrano, E.; Pradel, P.; Jailler, R.; Dubroeucq, H.; Bauchart, D. and Hocquette, J. F. 2007. Young salers suckled bull production: effect of diet on performance, carcass and muscle characteristics and meat quality. J Anim Sci, 1: 1068-1079.

Shackelford, S.D.; Wheeler, T.L.; Meade, M.K.; Reagan, J.O.; Byrnes, B.L. and Koohmaraie, M. 2001. Consumer impressions of tender select beef. J Anim Sci, 79: 2605-2614.

Shibata, M.; Matsumoto, K.; Hikino Y. and Yamamoto, N. 2014. Effect of indoor concentrate feeding vs. outdoor grazing on the expression 
of genes involved in muscle growth and nutrient content in japanese black steer muscle. Open J Anim Sci, 4: 297-304.

Silva, C.C.G.; Rego, O.A.; Simões, E.R.E. and Rosa, H.J.D. 2010. Consumption of high energy maize diets is associated with increased soluble collagen in muscle of Holstein bulls. Meat Sci, 86: 753-757.

Smith, G.C.; Belk, K.E.; Sofos, J.N.; Tatum, J.D. and Williams. S.N. 2000. Economic implications of improved coior stability in beef. Antioxidants in muscle foods: Nutritional strategies to improve quality. Wiley. New York, NY. pp. 397-426.

Solomon, M.B. and Lynch, G.P. 1988. Biochemical, histochemical and palatability characteristics of young ram lambs as affected by diet and electrical stimulation. J Anim Sci, 66: 1955-1962.

Soria, L.A. y Corva P.M. 2004. Factores genéticos y ambientales que determinan la terneza de la carne bovina. Arch Latinoam Prod Anim, 12: 73-88.

Stolowski, G.D.; Baird, B.E.; Miller, R.K. Savell, J.W.; Sams, A.R.; Taylor, J.F. Sanders, J.O. and Smith, S.B. 2006. Factors influencing the variation in tenderness of seven major beef muscles from three Angus and Brahman breed crosses. Meat Sci, 69: 215-224.

Swanek, S.S.; Morgan, J.B.; Owens, F.N.; Gill, D.R.; Strasia, C.A.; Dolezal, H.G. and Ray, F. K. 1999. Vitamin D3 supplementation of beef steers increases longissimus tenderness. J Anim Sci, 77: 874-881.

Sylvestre, M.N.; Balcerzak, D.; Feidt, C.; Baracos, V.E. and Bellut, J.B. 2002. Elevated rate of collagen solubilization and post mortem degradation in muscles of lambs with high growth rates: Possible relationship with activity of matrix metalloproteinases. J Anim Sci, 80: 1871-1878.

Therkildsen, M.; Houbak, M.B. and Byrne, D.V. 2008. Feeding strategy for improving tenderness has opposite effects in two different muscles. Meat Sci, 80:1037-1045.
Thompson, J.M. 2002. Managing meat tenderness. Meat Sci, 62: 295-308.

Troy, D.J. and Kerry, J.P. 2010. Consumer perception and the role of science in the meat industry. Meat Sci, 86: 214-226.

Tudor, G.D.; Coupar, F.J. and Pethick, D.W. 1996. Effect of silage diets on glycogen concentration in the muscle of yearling cattle. Proceedings of the Australian Society of Animal Production. p. 45.

Veiseth-Kent, E.; Hollung, K.; Ofstad, R.; Aass, L. and Hildrum K.I. 2010. Relationship between muscle microstructure, the calpain system, and shear force in bovine Longissimus dorsi muscle. J Anim Sci, 88: 3445-3451.

Vestergaard, M.; Oksbjerg, N. and Henckel, P. 2000. Influence of feed intensity, grazing and finishing feeding on muscle fibre characteristics and meat colour of Semitendinosus, Longissimus dorsi and Supraspinatus muscles of young bulls. Meat Sci, 54:177-185.

Weaver, A.D.; Bowker B.C. and Gerrard, D.E. 2008. Sarcomere length influences postmortem proteolysis of excised bovine semitendinosus muscle. J Anim Sci, 86: 1925-1932.

Wilkerson, E. 2016. The Influence of diet and oxidation on calcium retention of the mitochondria in Fresh beef. Thesis. University of Nebraska. Lincoln.

Wood, J.D.; Enser, M.; Fisher, A.V.; Nute, G.R.; Richardson, R.I. and Sheard, P.R. 1999. Manipulating meat quality and composition. Proc Nutrition Society, 58: 363-370. Nute, G.R.; Sheard, P.R.; Richardson, R.I.; Hughes, S.I. and Whittington, F.M. 2008. Fat deposition, fatty acid composition and meat quality: A review. Meat Sci, 78: 343-58. 